\section{Revisión de los aspectos éticos y criterios de bioseguridad en odontología en el contexto de la pandemia por COVID-19}

\author{
Review of the ethical issues and biosafety \\ measures in dentistry in the context of the \\ COVID-19 pandemic
}

\begin{abstract}
Resumen
El presente trabajo es una recopilación de información de bases de datos Medline, PubMed, ScienceDirect, Google Scholar y SciELO. El objetivo fue recopilar recomendaciones de bioseguridad planteadas a nivel nacional e internacional, resaltando el uso del consentimiento informado en la práctica odontológica en el contexto de la pandemia por COVID-19 y realizar un análisis de los aspectos éticos que involucra la práctica profesional. Se incluyeron artículos de revisión sistemática, manuales, entrevistas, editoriales, páginas web y literatura gris, se discriminó la evidencia y refrendó la información con artículos publicados en revistas indexadas, relacionando los aspectos éticos y deontológicos de la práctica odontológica y criterios de bioseguridad. Se concluye que es necesario el uso del consentimiento informado, por el alto riesgo de infección cruzada, así como el refuerzo de varios aspectos de bioseguridad como el uso adecuado del equipo de protección personal (EPP), en beneficio de los pacientes y del personal.
\end{abstract}

Palabras clave: Ética odontológica; Contención de riesgos biológicos; Odontología; Coronavirus (fuente: DeCS BIREME).

\begin{abstract}
This review compiles information from Medline, PubMed, ScienceDirect, Google Scholar, SciELO databases. Its objective is to provide biosecurity recommendations, highlighting the use of informed consent in dental practice in the context of the COVID-19 pandemic, according to current Peruvian regulations. A systematic review of articles, manuals, interviews, editorials, web pages, grey literature included, evidence discredited and information endorsed with articles published in indexed journals, relating ethical and deontological aspects of dental practice in biosafety criteria. In conclusion, the use of informed consent is important because there is a high risk of cross-infection as the reinforcement of several aspects of biosafety like the appropriate use of personal protective equipment (PPE), for the benefit of patients and staff.
\end{abstract}

Keywords: Dental ethics; Biohazard containment; Dentistry; Coronavirus (source: MeSH NLM).

\section{Artículo de Revisión}

\author{
José Antonio Alanya-Ricalde ${ }^{1, a}$, Monica Yizely \\ Llanos-Carazas $^{2, b}$, Sheila Acurio-Medina ${ }^{1, c}$ \\ ${ }^{1}$ Universidad Andina del Cusco, Cusco, Perú. \\ 2 Universidad Nacional Mayor de San Marcos, Lima, \\ Perú. \\ a Maestro en Docencia Universitaria. \\ ${ }^{\mathrm{b}}$ Magister en Docencia e Investigación en Salud. \\ c Estudiante de Estomatología.
}

\section{Correspondencia:}

José Antonio Alanya-Ricalde: jalanya@uandina.edu.pe Urb. Ttio, pasaje Miraflores Y-1-27, 40108007 Wanchaq - Cusco

ORCID: 0000-0003-4752-9983

\section{Coautores:}

Monica Yizely Llanos-Carazas: mllanosc1@unmsm.edu.pe ORCID: 0000-0002-3179-8751

Sheila Acurio-Medina: sheilaacurio@gmail.com

ORCID: 0000-0002-8620-9619

Editor:

Juan Carlos Cuevas-González

Universidad Autónoma de Ciudad Juárez, México.

Conflicto de intereses: los autores declaran no tener conflictos de interés.

Fuente de financiamiento: autofinanciado.

Recibido: 03/12/20

Aceptado: $26 / 02 / 21$

Publicado: 01/07/21 


\section{Introducción}

La bioseguridad y la ética son aspectos importantes para el desempeño del cirujano dentista. El primero va referido a la parte técnica y el segundo está directamente relacionado con la responsabilidad y moral en el trabajo, esto repercute en la práctica profesional, en la salud del cirujano dentista, así como en la protección del paciente, disminuyendo la contaminación cruzada, bacteriana y la propagación del virus SARS-CoV-2 (COVID -19), el cual representa un alto riesgo alto de exposición ${ }^{1}$.

La protección del paciente debe ser minuciosamente contemplada, ya que el ambiente odontológico es un entorno de alto riesgo por situación de exposición, porque se genera aerosol durante la atención dental ${ }^{1-3}$. Los odontólogos trabajan directamente en la cavidad bucal, siendo éste reservorio potencial para la transmisión de COVID-19. En este sentido se deben tomar en cuenta medidas de protección y protocolos de bioseguridad, además de una serie de aspectos éticos antes, durante y después de la atención odontológica.

Las medidas de bioseguridad y aspectos éticos consideradas en la presente revisión son las concernientes a la protección del paciente, del profesional así como también los cuidados del ambiente odontológico, la desinfección de superficies, de materiales y equipos, el cual debe ser minucioso para cada ${ }^{1,4}$.

La Asociación Latinoamericana de Odontopediatría 5,6, ha desarrollado en el presente año dos manuales acerca de la evaluación pre-atención y atención odontológica apropiada durante la pandemia COVID-19 en niños, optimizando la calidad de atención y minimizando el riesgo de transmisión de coronavirus entre pacientes y el equipo odontológico que realiza el tratamiento.

En una entrevista el Doctor Herazo ${ }^{7}$ indica que "El principio ético universal de hacer siempre bien y nunca el mal es algo que todos los seres humanos deben aplicar" esto en términos más formales se refiere a la beneficencia y no maleficencia de la práctica clínica en odontología y que los odontólogos están muy comprometidos y obligados con el cumplimiento de rigurosos protocolos de asepsia, antisepsia y bioseguridad en su cuerpo, en sus consultorios o clínicas, instrumental, materiales, uniformes y productos desechables, como también con la máxima higiene de sus auxiliares, para así evitar el contagio y la expansión del COVID-19, debido a que el profesional está expuesto al contacto con sangre y específicamente, con saliva y piel de la cara de los pacientes.

Pérez-Domínguez ${ }^{8}$ indicó que es necesario el diseño de protocolos estrictos de bioseguridad que disminuyan los riesgos de contagio, con el criterio de que todo paciente atendido es un potencial caso positivo de COVID-19 ${ }^{1,9}$, debido a la baja o inexistente disponibilidad de pruebas de laboratorio para detectar la infección previa a la consulta, puesto que pacientes aparentemente sanos pueden formar parte de casos asintomáticos o pre-sintomáticos con alta carga viral.
Sigua-Rodríguez ${ }^{10}$ proporciona una actualización y una serie de recomendaciones de todos los cuidados específicos que existen hasta hoy, recordando que estas informaciones pueden sufrir cambios asociados a las actualizaciones y reglamentos con posibles nuevas informaciones científicas y cambios legislativos, estas adaptaciones serán realizadas según la necesidad en cada región, ya que nos enfrentamos a un virus que cada día nos sorprende más.

En la actualidad se vive la segunda ola de casos de COVID-19 en muchos países de Europa tal es el caso de la Reino Unido en el cual se detectó una nueva cepa, Esta variante se denomina SARS-CoV-2 VUI 202012/01 (por las siglas en inglés de «variante en investigación, año 2020, mes 12 , variante 01 , siendo que esta variante puede propagarse más fácilmente entre las personas de acuerdo a estudios preliminares ${ }^{11}$, por lo cual es recomendable que el profesional se mantenga actualizado sobre la enfermedad y sus diferentes vías de contagio, con el objetivo de que los cirujanos dentistas brinden asistencia odontológica durante las fases críticas de la pandemia.

Es por ello, que el presente trabajo tiene por objetivo recopilar recomendaciones de bioseguridad planteadas a nivel nacional e internacional, resaltando el uso del consentimiento informado en la práctica odontológica en el contexto de la pandemia por COVID-19, en relación a la normativa vigente peruana emitida por el Ministerio de Salud (MINSA); y realizar un análisis de los aspectos éticos que involucra la práctica profesional.

\section{Materiales y métodos}

El presente trabajo de investigación es una recopilación de varios artículos de revisión, manuales, entrevistas, editoriales que abarcan temas de bioseguridad en la atención odontológica y los argumentos éticos validos en el ejercicio de la profesión. En vista que el presente análisis bibliográfico basa su búsqueda en hechos recientes como la pandemia del COVID-19, los artículos revisados son de data de enero a diciembre del 2020 y artículos presentados con 5 años de antigüedad como máximo.

Se empleó el método cualitativo y con análisis de contenido de tipo directo. La búsqueda de la evidencia científica fue obtenida principalmente a través de los siguientes buscadores: MEDLINE (National Library of Medicine, USA - NLM) y su portal PubMed, Science Direct, SciELO. En los cuales se utilizó los descriptores: Éética, bioseguridad, odontología y COVID-19, del mismo modo y en base a la evidencia encontrada se tuvo que utilizar páginas web oficiales de la OMS, OPS, MINSA y sociedades iberoamericanas de odontología, páginas web de Organismo de Salud de otros países y literatura gris, de la cual se discriminó la evidencia y refrendó la información con artículos publicados en revistas indexadas.

\section{Antecedentes de los aspectos éticos y bioseguridad a causa del virus COVID-19}

En Wuhan una ciudad de la provincia de Hubei en China, en el mes de diciembre del 2019, surgió un nuevo 
brote de neumonía por el nuevo coronavirus COVID-19, siendo declarado pandemia en marzo de 2020, desde entonces se ha registrado una rápida propagación a escala comunitaria, regional e internacional, con un aumento exponencial del número de casos y muertes. A nivel mundial, hasta las 5:20 pm CET, 6 de enero de 2021, se han notificado a la OMS 85091012 casos confirmados de COVID-19, incluidas 1861005 muertes ${ }^{12}$.

Desde sus inicios hasta el día de hoy el mundo ha cambiado; tanto en el estilo de vida, poniendo a prueba los sistemas sanitarios, donde la atención odontológica y otras actividades económicas se han suspendido durante varios meses en todos los países en presencia de la enfermedad. El Perú es uno de los países más afectados en Latinoamérica según declaraciones de los especialistas ${ }^{13}$. El gobierno peruano fue uno de los primeros de Latinoamérica en declarar el Estado de Emergencia Nacional por las graves circunstancias que afectan la vida de la Nación a consecuencia del brote del COVID-19, a través del Decreto Supremo No 044-2020-PCM publicado en el Diario oficial el peruano el 15 de marzo del 2020. En el cual se ordenó el confinamiento obligatorio y el cese temporal de todas las actividades económicas en vista de que puede afectar la salud y vida de las personas ${ }^{14}$, este ha sido modificado por varios decretos supremos hasta la actualidad ampliando y extendiendo las medidas restrictivas y dando lineamientos de apertura de las diferentes actividades económicas de manera escalonada.

La atención odontológica ha sufrido durante este periodo de cuarentena, la restricción de las atenciones limitándolas a la atención de urgencias y emergencias debido a los reportes de exposición, transmisión y el alto riesgo que representa para la propagación del COVID-19 ${ }^{15}$, en este sentido las instituciones pertinentes han desarrollado una serie de recomendaciones, directivas y protocolos, acerca de la atención de pacientes considerándolos como portadores sospechosos de SARS-CoV-2 1,9. Por ejemplo, en Perú se decretó la directiva sanitaria $\mathrm{N}^{\circ} 100$ que reglamenta la atención odontológica en el contexto de la pandemia del COVID-19 ', que contempla todos los procedimientos a seguir en cuanto a métodos de bioseguridad y protección antes, durante y después de la atención odontológica; sin embargo se ve con preocupación el incremento de los casos de odontólogos con COVID-19, comprobando así una falla en la aplicación de la normativa o aparente desconocimiento de los aspectos rigurosos y éticos que debe contemplar la práctica clínica.

En este sentido podemos mencionar algunos aspectos importantes relacionados a atención odontológica, vinculados a la parte de aplicación ética de estos procedimientos: como la asepsia, antisepsia, desinfección y esterilización de materiales, instrumental e indumentaria, en beneficio del paciente y protección del profesional.

\section{Medidas de bioseguridad}

Referidas al paciente. Las medidas de bioseguridad concernientes al paciente son procedimientos que concuerdan con la ética deontológica en favor de las personas que requieren atención odontológica, en su beneficio y justificado en casos de urgencia; este integra la desinfección al ingreso del consultorio dental, así como: toda la indumentaria del paciente, colocación del mandil quirúrgico descartable, cubre zapatos, gorra descartable y lentes de protección ${ }^{1,2}$.

El motivo por el imperativo uso del EPP por parte del paciente radica en la posibilidad de que el virus se pueda alojar en la ropa del paciente y este llevarlo a la casa, esto también lo manifiestan diversos autores en las recomendaciones en la atención odontológica ${ }^{16-22}$. Al realizar la revisión bibliográfica correspondiente no manifiesta de manera clara el uso de equipo de protección para el paciente creándose un vacío en los diversos protocolos.

De la misma manera el lavado de manos durante 40 segundos y el empleo del gel hidroalcohólico ${ }^{1,2,19,23}$.

Finalmente el uso de enjuagues bucales antimicrobianos preoperatorios los cuales se han recomendado a fin de reducir el número de microorganismos en aerosoles y gotas durante procedimientos orales ${ }^{24}$, pudiendo ser:

- Peróxido de hidrógeno al 1\% durante 30 segundos1 minuto.

- Enjuague bucal con gluconato de clorhexidina al $0,12 \%{ }^{1}$.

- Povidona yodada al $0,5 \%$ al $1 \%$ durante un minuto ${ }^{25}$.

- Cloruro de cetilpiridinio al 0,05\% - 0,1\% ${ }^{26}$.

En un ensayo clínico realizado en Singapur ${ }^{27}$, se demostró la efectividad aunque no de manera concluyente, de estos enjuagues en la reducción de SARS-CoV-2 en saliva de pacientes con COVID-19.

Así mismo es importante considerar el tiempo entre paciente y paciente de aproximadamente 30 minutos ${ }^{23}$.

Referidos al cirujano dentista. Otro aspecto ético deontológico contempla la protección del odontólogo de manera completa, protegiendo ojos, boca y manos para evitar contacto directo con el paciente y el aerosol que se produzca, evitando así una contaminación cruzada.

Equipo de protección personal (EPP): contempla un valor moral y ético muy importante en el cual se manifiesta el beneficio que se obtiene al utilizar esta indumentaria ${ }^{28}$, involucra protección y salud a las personas con riesgo ocupacional ya que es imposible mantener distanciamiento porque se trabaja a menos de un metro de distancia y cuyos procedimientos generan aerosol 1,19,23, el equipo consta de: respirador, protector facial, guantes y bata quirúrgica, se recomienda que sea de uso descartable y de uso exclusivo para cada paciente.

El respirador indicado es el de tipo "N95" según US NIOSH, o "FFP2" según EN 149N95 ${ }^{19}$. También se debe emplear protector facial que permita una adecuada desinfección, visibilidad e idealmente antiempañante, que cubra completamente los lados y la longitud de la cara. El uso de guantes y bata quirúrgica descartable. ${ }^{1}$ 
Las recomendaciones para garantizar la calidad y control de infecciones por SARS-CoV-2 en la atención dental se enfocan en la disminución en la generación de aerosoles, para lo cual es imprescindible el uso de mascarillas o respiradores, el uso del suctor de saliva, suctor extra oral, así como el uso de aislamiento absoluto ${ }^{15}$, procurando anular el uso de las escupideras, estas prácticas se reflejan en la beneficencia y no maleficencia en cuanto a la ética referida a la bioseguridad.

La disponibilidad, escases y el alto costo de las mascarillas llamadas también respiradores se ha visto afectada en el actual contexto, con el precepto que los equipos de protección deberían ser de un solo uso, atendiendo el indicador de que todo paciente es sospechoso de COVID-19, sin embargo en la actualidad se observa la utilización de respiradores más allá de la vida útil designada por el fabricante, el uso de los respiradores durante largos periodos excediendo a las normas sugeridas por la OMS de 6 horas. Así como el reacondicionamiento y la posterior reutilización de los EPP. La reutilización de estas barreras implica que se tiene que realizar un proceso de reacondicionamiento lo cual es riesgoso para el personal que lo realiza y contribuye a que se produzca contagio en consultorios que prestan servicios a bajos costos y sin las medidas adecuadas, atentando contra la salud propia y de los que se atienden ${ }^{29}$.

\section{Consentimiento informado}

El consentimiento informado es un documento que guarda las pautas éticas de autonomía en el tratamiento de paciente odontológico asi lo contempla Bravo ${ }^{30}$ en su análisis de la reglamentación en Chile, del mismo modo el Código de Ética y Deontología del Perú, ${ }^{31}$ que obliga al odontólogo en el uso de este.

Para la Asociación Dental Americana (ADA) ${ }^{32}$, en cualquier momento, no solo en contexto de pandemia, el consentimiento informado de cualquier procedimiento es más que una mera firma en el formulario: es el resultado final del diálogo entre el odontólogo y el paciente.

Existen publicaciones ${ }^{33,34}$ que indican el uso de este proceso, en el contexto actual donde manifiestan que el paciente debe ser consiente de los riesgos de transmisión del COVID-19 que se presentan en la atención odontológica, inclusive aplicando todos los métodos de bioseguridad por la generación de aerosoles que implica la práctica odontológica ${ }^{1}$.

En el marco de pandemia se ha recomendado a nivel nacional y en otros países que la atención en odontología sea solo para tratamientos en caso de urgencia y emergencia por lo cual se ha reglamentado el uso de consentimiento informado para estos casos por citar algunos ejemplos, por el Colegio Oficial de Dentistas en Brasil asi como la directiva sanitaria $\mathrm{N}^{\circ} 100 / \mathrm{MINSA} / 2020 /$ DGIESP del Ministerio de Salud del Perú ${ }^{1}$ del mismo modo en otros países ${ }^{35,36}$.

Lamentablemente es muy probable que el uso de este recurso tan importante en la práctica odontológica, como base de la documentación legal y deontológica no se esté utilizando, en el caso de la realidad peruana, las urgencias estomatológicas no se atienden en establecimientos del estado, porque el protocolo de atención en el Perú no incluye la presencia de un estomatólogo en los servicios de emergencia ${ }^{37}$, este aspecto es cubierto por la práctica privada, sin embargo no se presentan trabajos relacionados a la aplicación del consentimiento en este país y en el extranjero, por lo que es imperativo su revisión y es recomendable realizar estudios acerca de la importancia que le están dando los odontólogos en Latinoamérica y en el mundo.

\section{Conclusiones}

Las medidas de bioseguridad que se realizan en la práctica odontológica son muy importantes para reducir la trasmisión de COVID-19, contempla aspectos éticos deontológicos los cuales van en beneficio del paciente, del profesional y personal auxiliar, una práctica odontológica de manera responsable es importante para contribuir a la reducción de contaminación cruzada en este ámbito.

Se debe reforzar el hábito de utilizar el consentimiento informado en el contexto del COVID-19, puesto que se cumple con el principio ético de autonomía, el cual indica que a pesar que la situación se presenta como riesgosa, expresa la voluntariedad del paciente de someterse a los procedimientos que sean necesarios. Del mismo modo, la periodicidad y minuciosidad de los procesos de asepsia, antisepsia y desinfección de ambientes y equipo asi como de instrumental y material de uso odontológico, en beneficio del paciente odontológico. Finalmente minimizar la atención solo a casos de urgencia el cual cumple con el principio de justicia de manera clara.

\section{Referencias bibliográficas}

1. Resolución Ministerial $\mathrm{N}^{\circ}$ 288-2020-MINSA que aprueba la Directiva Sanitaria N ${ }^{\circ}$ 100/MINSA/2020/ DGIESP. Lima: Ministerio de Salud - MINSA - Perú; 2020. Disponible en: https://www.gob.pe/institucion/ minsa/normas-legales/578465-288-2020-minsa

2. Barabari P, Moharamzadeh K. Novel Coronavirus (COVID-19) and Dentistry-A Comprehensive Review of Literature. Dent J [Internet]. 2020 [citado el 22 de julio de 2020];8(2). Disponible en: https://www.ncbi. nlm.nih.gov/pmc/articles/PMC7345990/ doi: 10.3390/ dj8020053

3. Sabino-Silva R, Jardim ACG, Siqueira WL. Coronavirus COVID-19 impacts to dentistry and potential salivary diagnosis. Clin Oral Investig [Internet]. el 20 de febrero de 2020 [citado el 22 de julio de 2020];1-3. Disponible en: https://www.ncbi.nlm.nih.gov/pmc/articles/ PMC7088419/ doi: 10.1007/s00784-020-03248-x

4. Resolución Ministerial $N^{\circ}$ 1472-2002-SA-DM que aprueba el Manual de Desinfección y Esterilización Hospitalaria. Lima: Ministerio de Salud - MINSA - Perú; 2002. [citado el 11 de enero de 2021] Disponible en: https://www.gob.pe/institucion/minsa/normas-legales/254621-1472-2002-sa-dm 
5. Asociación Latinoamericana de Odontopediatría. Ruta de atención para procedimientos de Odontología Pediátrica durante la etapa de confinamiento o cuarentena de la pandemia COVID-19. Rev Latinoam Odontop [Internet]. 2020 [citado el 5 de mayo de 2020]; 10(2). Disponible en: https://www.revistaodontopediatria.org/

6. Asociación Latinoamericana de Odontopediatría. Tratamiento de caries en época de COVID-19: Protocolos clínicos para el control de generación de aerosoles. Rev Latinoam Odontop [Internet]. 2020 [citado el 5 de mayo de 2020];10(2). Disponible en: https://www.revistaodontopediatria.org/ediciones/2020/2/art-2/

7. Herazo BA. La ética en el manejo del Covid-19 [Internet]. Dental Tribune. 2020 [citado el 5 de mayo de 2020]. Disponible en: https://la.dental-tribune.com/news/el-odontologo-debe-apoyar-siempre-al-paciente-2-2/

8. Pérez-Domínguez M, Pérez-Ybarra L. SARS-CoV-2 en saliva: potencial vía de contagio e implicaciones en el tratamiento del paciente odontológico / SARS-CoV-2 in saliva: potential route of infection and implications in the treatment of dental patient. Odous Científica. 2020;21(1):77-88.

9. Souza RCC, Costa PS, Costa LR. Precauçóes e Recomendaçóes sobre Sedação Odontológica durante a Pandemia de COVID-19. Rev Bras Odontol [Internet]. 2020 [citado el 5 de mayo de 2020]; 77(1788):3. Disponible en: http://cro-df.org.br/pdf/artigocorona.pdf doi: http://dx. doi.org/10.18363/rbo.v77.2020.e1788

10. Sigua-Rodríguez EA, Bernal-Pérez JL, Lanata-Flores AG, Sánchez C, Chessa JR, Haidar Z, et al. COVID-19 y la Odontología: una Revisión de las Recomendaciones y Perspectivas para Latinoamérica. Int J Odontostomat. 2020;14(3):299-309.

11. Organización Mundial de la Salud - OMS (WHO eng.). OMS | Cepa variante del SARS-CoV-2 - Reino Unido [Internet]. WHO. World Health Organization; 2020 [citado el 11 de enero de 2021]. Disponible en: http:// www.who.int/csr/don/21-december-2020-sars-cov2-variant-united-kingdom/es/

12. World Health Organization. WHO Coronavirus Disease (COVID-19) Dashboard | WHO Coronavirus Disease (COVID-19) Dashboard [Internet]. WHO Coronavirus Disease (COVID-19) Dashboard. 2021 [citado el 11 de enero de 2021]. Disponible en: https://covid19.who.int/

13. Arredondo A. OPS: Latinoamérica es el "epicentro" de la pandemia del COVID-19| Voice of America - Spanish [Internet]. Portal de Noticias. 2020 [citado el 9 de julio de 2020]. Disponible en: https://www.voanoticias.com/ salud/ops-latinoamerica-epicentro-pandemia

14. Decreto Supremo. D.S. N ${ }^{\circ}$ 044-2020-PCM que declara Estado de Emergencia Nacional por las graves circunstancias que afectan la vida de la Nación a consecuencia del brote del COVID-19. Lima: Diario Oficial del Bicentenario El Peruano; 2020. [citado el 11 de mayo de 2020] Disponible en: http://busquedas. elperuano.pe/normaslegales/decreto-supremo-que-declara-estado-de-emergencia-nacional-po-decreto-supremo-n-044-2020-pcm-1864948-2/

15. Baghizadeh Fini M. What dentists need to know about COVID-19. Oral Oncol [Internet]. junio de 2020 [citado el 9 de julio de 2020];105:104741.
Disponible en: https://www.ncbi.nlm.nih.gov/pmc/ articles/PMC7186204/ DOI: 10.1016/j.oraloncology.2020.104741

16. Gurzawska-Comis K, Becker K, Brunello G, Gurzawska A, Schwarz F. Recommendations for Dental Care during COVID-19 Pandemic. J Clin Med. 2020;9(6):18-33. DOI: https://doi.org/10.3390/jcm9061833

17. Ge Z-Y, Yang L-M, Xia J-J, Fu X-H, Zhang Y-Z. Possible aerosol transmission of COVID-19 and special precautions in dentistry. J Zhejiang Univ Sci B. 2020;21(5):361-8. DOI: 10.1631/jzus.B2010010

18. Tuñas IT de C, Silva ET da, Santiago SBS, Maia KD, Silva-Júnior GO. Doença pelo Coronavírus 2019 (COVID-19): Uma abordagem preventiva para Odontologia. Rev Bras Odontol [Internet]. 2020 [citado el 5 de mayo de 2020];77(0):1-7. Disponible en: http://revista.aborj. org.br/index.php/rbo/article/view/1776 DOI: http://dx. doi.org/10.18363/rbo.v77.2020.e1776

19. Meng L, Hua F, Bian Z. Coronavirus Disease 2019 (COVID-19): Emerging and Future Challenges for Dental and Oral Medicine. J Dent Res [Internet]. 2020 [citado el 5 de mayo de 2020]; Disponible en: https:// www.ncbi.nlm.nih.gov/pmc/articles/PMC7140973/ DOI: $10.1177 / 0022034520914246$

20. Morales Navarro D. Acciones del personal de salud del área estomatológica en relación al COVID-19. Rev Cuba Estomatol [Internet]. 2020 [citado el 16 de mayo de 2020];57(1):3245. Disponible en: http://revestomatologia.sld.cu/index.php/est/article/view/3245

21. Zhiyong L, Liuyan M. Prevention and control of novel coronavirus infection in department of stomatology. Chin J Stomatol [Internet]. 2020 [citado el 5 de mayo de 2020];55(04):217-22. Disponible en: http://rs.yiigle. com/CN112144202004/1188491.htm doi: 10.3760 / cma.j.cn112144-20200210-00044

22. Spagnuolo G, De Vito D, Rengo S, Tatullo M. COVID-19 Outbreak: An Overview on Dentistry. Int J Environ Res Public Health [Internet]. 2020 [citado el 5 de mayo de 2020];17(6). Disponible en: https:// www.ncbi.nlm.nih.gov/pmc/articles/PMC7143628/ DOI: $10.3390 /$ ijerph17062094

23. Federación de sociedades científicas de especialidades odontológicas de Chile FESODECH. Protocolo atención odontológico post COVID-19 [Internet]. 2020. [citado el 11 de enero de 2021] Disponible en: https://odontopediatria.cl/wp-content/uploads/2020/05/Protocolo-de-atencio\%CC\%81n-Covid19-FESODECH-1.pdf

24. Vergara-Buenaventura A, Castro-Ruiz C. Use of mouthwashes against COVID-19 in dentistry. $\mathrm{Br} \mathrm{J}$ Oral Maxillofac Surg. octubre de 2020;58(8):924-7. DOI: 10.1016/j.bjoms.2020.08.016

25. Burton MJ, Clarkson JE, Goulao B, Glenny A-M, McBain AJ, Schilder AG, et al. Antimicrobial mouthwashes (gargling) and nasal sprays administered to patients with suspected or confirmed COVID-19 infection to improve patient outcomes and to protect healthcare workers treating them. Cochrane Database Syst Rev [Internet]. 2020 [citado el 11 de enero de 2021];(9). Disponible en: https://www.cochranelibrary.com/cdsr/ doi/10.1002/14651858.CD013627.pub2/full/es doi: https://doi.org/10.1002/14651858.CD013627.pub2 
26. Guiñez CM. Impacto del COVID-19 (SARS-CoV-2) a Nivel Mundial, Implicancias y Medidas Preventivas en la Práctica Dental y sus Consecuencias Psicológicas en los Pacientes. Int J Odontostomatol [Internet]. 2020; 14(3):271-8. [citado el 11 de enero de 2021]; Disponible en: http://www.ijodontostomatology.com/es/articulo/ impacto-del-covid-19-sars-cov-2-a-nivel-mundial-implicancias-y-medidas-preventivas-en-la-practica-dental-y-sus-consecuencias-psicologicas-en-los-pacientes/

27. Seneviratne CJ, Balan P, Ko KKK, Udawatte NS, Lai $\mathrm{D}, \mathrm{Ng}$ DHL, et al. Efficacy of commercial mouth-rinses on SARS-CoV-2 viral load in saliva: randomized control trial in Singapore. Infection [Internet]. 2020;1-7. [citado el 11 de enero de 2021] Disponible en: https:// www.ncbi.nlm.nih.gov/pmc/articles/PMC7734110/ DOI: $10.1007 / s 15010-020-01563-9$

28. Ethics Subcommittee of the Council on Ethics, Bylaws and Judicial Affairs. Ethical practice during the COVID-19 pandemic. J Am Dent Assoc 1939. 2020;151(5):377-8. DOI: 10.1016/j.adaj.2020.03.038

29. Organización Panamericana de la Salud OPS. Aspectos técnicos y regulatorios sobre el uso prolongado, la reutilización y el reprocesamiento de respiradores en períodos de escasez. 10 de junio del 2020 [Internet]. OPS; 2020 [citado el 12 de enero de 2021]. Disponible en: https:// iris.paho.org/handle/10665.2/52460.

30. Bravo-R. L, Lagos-Tissie D, Bravo-R. L, Lagos-Tissie D. Nuevo Código de Ética Profesional Odontológica en Chile: Contribución para una Relación Médico-Paciente Moderna. Int J Odontostomatol [Internet]. 2017; 11(4):419-24. [Citado el 9 de julio de 2020] Disponible en: https://scielo.conicyt.cl/scielo.php?script=sci_abstract\&pid=S0718-381X2017000400419\&lng=es\&nrm=iso\&tlng=en DOI: http://dx.doi.org/10.4067/ S0718-381X2017000400419.

31. Colegio Odontológico del Perú. Código de ética y deontología [Internet]. 2016. [citado el 9 de julio de 2020] Disponible en: https://www.cop.org.pe/wp-content/ uploads/2016/08/CODIGO-DE-ETICA-Y-DEONTOLOGIA-2016-1.pdf
32. American Dental Asociation (ADA eng). Preguntas frecuentes sobre COVID-19 [Internet]. ADA Center for professional success. 2020 [citado el 12 de enero de 2021]. Disponible en: https://success.ada.org/en/practice-management/patients/coronavirus-frequently-asked-questions\#patientcommunication

33. Seoane Campomar M. Asistencia en Prostodoncia Removible frente a la pandemia por COVID-19. Odontoestomatología. 2020;22:79-94. DOI: http://dx.doi. org/10.22592/ode2020nespa8

34. Matus-Abásolo CP, Nemeth-Kohanszky ME, Inostroza-Tapia MA. Atención de Pacientes en Tratamiento de Ortodoncia Durante la Pandemia COVID-19 (SARS-CoV-2). Presentación de un Algoritmo. Int J Odontostomatol [Internet]. diciembre de 2020 [citado el 12 de enero de 2021];14(4):489-94. Disponible en: https://scielo.conicyt.cl/scielo.php?script=sci_abstract\&pid=S0718-381X2020000400489\&lng=es\& $\&$ nrm =iso\&tlng=es DOI: http://dx.doi.org/10.4067/S0718381X2020000400489.

35. Kornecki Radzinski F. COVID 19 y la Cirugía Bucomaxilofacial. Odontoestomatología. 2020;22:50-9. DOI: http://dx.doi.org/10.22592/ode2020nespa5

36. Ministerio de Salud Pública - Ecuador. Protocolo para la atención odontológica durante la emergencia sanitaria por Covid-19 [Internet]. 2020. [citado el 10 de julio de 2020] Disponible en: https://www.salud.gob.ec/wp-content/uploads/2020/06/PROTOCOLO-PARA-ATENCION-ODONTOLOGICA-DURANTE-LA-EMERGENCIA-SANITARIA-POR-COVID.pdf

37. Quincho-Rosales DA, Castro-Rodríguez Y, Grados-Pomarino S, Quincho-Rosales DA, Castro-Rodríguez Y, Grados-Pomarino S. Consideraciones sobre la atención estomatológica en el Perú durante la pandemia por la COVID-19. Rev Cuba Estomatol [Internet]. 2020; 57(3). [Citado el 12 de enero de 2021] Disponible en: http://scielo.sld.cu/scielo.php?script=sci_abstract\&pi$\mathrm{d}=$ S0034-75072020000300005\&lng=es\&nrm=iso\&tl$\mathrm{ng}=\mathrm{es}$ 